\title{
Inverse Design of Single- and Multi-Rotor Horizontal Axis Wind Turbine Blades using Computational Fluid Dynamics
}

\author{
Behnam Moghadassian and Anupam Sharma* \\ Department of Aerospace Engineering, Iowa State University, Ames, Iowa, \\ 50011 \\ * sharma@iastate.edu
}

\begin{abstract}
A method for inverse design of horizontal axis wind turbines (HAWTs) is presented in this paper. The direct solver for aerodynamic analysis solves the Reynolds Averaged Navier Stokes (RANS) equations, where the effect of the turbine rotor is modeled as momentum sources using the actuator disk model (ADM); this approach is referred to as RANS/ADM. The inverse problem is posed as follows: for a given selection of airfoils, the objective is to find the blade geometry (described as blade twist and chord distributions) which realizes the desired turbine aerodynamic performance at the design point; the desired performance is prescribed as angle of attack $(\alpha)$ and axial induction factor $(a)$ distributions along the blade. An iterative approach is used. An initial estimate of blade geometry is used with the direct solver (RANS/ADM) to obtain $\alpha$ and $a$. The differences between the calculated and desired values of $\alpha$ and $a$ are computed and a new estimate for the blade geometry (chord and twist) is obtained via nonlinear least squares regression using the Trust-Region-Reflective (TRF) method. This procedure is continued until the difference between the calculated and the desired values is within acceptable tolerance. The method is demonstrated for conventional, single-rotor HAWTs and then extended to multi-rotor, specifically dual-rotor wind turbines. The TRF method is also compared with the multi-dimensional Newton iteration method and found to provide better convergence when constraints are imposed in blade design, although faster convergence is obtained with the Newton method for unconstrained optimization.
\end{abstract}

\section{Introduction}

Rapid increase in utilization of turbines to harvest clean and renewable wind energy resource has introduced new challenges for researchers. As power production is directly dependent on the design of the wind turbine blades, considerable research studies have focused on developing methods to improve blade design in order to increase the output power. One approach to blade design is to use direct analysis codes and perform parametric sweeps to identify the highest-performing blade design. However, this approach is computationally demanding and does not guarantee that the optimum design will be reached 17]. More recently, researchers have started using optimization algorithms in the blade design process. For example, Chattot 6 used the Lagrange multiplier optimization method to maximize power while constraining thrust at a given tip speed ratio. The inputs to the optimization algorithm were blade twist and chord. A 
prescribed-wake vortex line method (VLM) was used that consisted of the Goldstein model 10 to analyze the trailing wake vortex structure behind the rotor blades and used the Biot-Savart formula to calculate the induction.

Inverse algorithms were introduced in wind turbine blade design process by Selig and Tangler [27. They combined the multi-dimensional Newton method with the Blade Element Momentum (BEM) theory to perform inverse blade design. This method is implemented in the software PROPID which can perform inverse design in a variety of ways. One way is to prescribe the desired distributions of axial induction factor $(a)$ and lift coefficient $\left(c_{l}\right)$ along the blade, and the inverse design gives the blade geometry (blade twist and chord distributions) that would yield the prescribed $a$ and $c_{l}$ distributions. The desired radial distributions of $a$ and $c_{l}$ are carefully selected based on prior experience of the designer but with the general aim of maximizing annual energy production (AEP). Any combination of aerodynamic quantities, e.g., angle of attack $(\alpha)$, lift coefficient $\left(c_{l}\right)$, circulation $(\Gamma)$, axial induction $(a)$, etc. can be prescribed as desired distributions that the final blade design is required to satisfy.

Lee 17] proposed the use of VLM over the BEM theory as the direct solver in inverse design of HAWT blades to model the effects of the three dimensional aerodynamic features of modern turbine blades, such as sweep, pre-bend, non-planar wing tips, etc. The VLM allows for partial coupling of the different radial sections of the blade through induced velocity computed via Biot Savart's law. Note that the coupling is partial because the method still uses 2-D airfoil polars and cannot account for spanwise flow over the blade. Radial distributions of $a$ and $c_{l}$ were used to prescribe the desired blade aerodynamics. The multi-dimensional Newton method was used to iterate on blade geometry (twist and chord) to obtain the prescribed $a$ and $c_{l}$ distributions.

Selig and Coverstone-Carroll 26] and Giguere and Selig [9] merged the optimization techniques with inverse design approaches to find the blade geometry that would maximize AEP. They utilized the BEM theory along with a genetic algorithm to reach their goal of maximizing AEP through blade design. If blade design is sought with the sole objective of maximizing the AEP without any constraints, the final blade design would have extremely high solidity inboard. The inboard $c_{l}$ and $a$ distributions are therefore tailored during inverse design procedure to yield a practical blade design that would satisfy design-, manufacturing-, and transportation constraints. Lee et al. 16 used this idea to determine the blade shape by considering a target function that includes the AEP as well as the costs for blade masters, mold sets, tooling and blade production. They used the strip theory 1$]$ to perform the inverse design, while their target was to minimize energy loss at the Betz optimum condition. The inverse blade design was a part of a global optimization algorithm which aimed to maximize power production and simultaneously minimize blade cost.

Aerodynamics models that solve the Navier-Stokes equations offer higher fidelity in analysis and design of wind turbine blades in comparison with models based on solving the simplified potential flow equations (e.g., BEM and VLM). The Navier-Stokes equations are usually solved with some simplifying assumptions. For wind turbine aerodynamics application, the incompressible turbulent flow (at least turbulence at high wavenumbers) is modeled (e.g., using eddy-viscosity based turbulence models), rather than directly solved. If the interest is only in mean quantities, time variation is ignored and Reynolds Averaged Navier-Stokes (RANS) equations are used with appropriate turbulence models that model the entire turbulence spectra. Many researchers 2, 3, 14, 18,28 have utilized different forms of the RANS model to investigate wind turbines aerodynamics. In one computationally efficient but simplified approach, the blade geometry is not resolved in the simulation, instead, the effect of the spinning blades on the air flow is introduced as source terms (typically as body forces) in the Navier-Stokes equations. 
The Actuator Line Method (ALM) 31] and the Actuator Disk Method (ADM) 29 are the most commonly used methods to simulate the effect of rotor blades using body forces without resolving the blade geometry. Both methods use look-up tables for 2-D lift and drag polars (obtained via prior experiments or simulations) with the local flow velocity vector to calculate the net sectional force at each radially discretized element of the blade. The net force is then applied as a spatially distributed (typically a Gaussian distribution is used) source around the radial element. Even though these methods use the strip theory approach with 2-D airfoil polars, they are still advantageous over potential flow methods in that they can, to some degree, simulate turbulent wake mixing. Inverse design approaches that use such CFD techniques then have the potential to explore designs that maximize wake mixing, and hence minimize wake losses in wind farms. This paper presents a methodology to use RANS CFD to perform inverse design of HAWT blades. The approach adopted here for inverse design is to specify the desired $\alpha$ and $a$ distributions and let the inverse design compute the blade twist and chord. The Trust-Region-Reflective method [4,7] is used as the optimization algorithm. The proposed inverse design procedure is verified for a number of conventional, single-rotor horizontal axis wind turbines.

The inverse design method is then extended to multi-rotor wind turbines, specifically the dual-rotor wind turbine (DRWT) proposed by Rosenberg et al. 24. Manufacturing and transportation constraints on rotor blades of conventional, utility scale wind turbines result in aerodynamically sub-optimal design in the blade root region (inner $25 \%$ ) 24. This results in inefficient extraction of energy by the rotor in this region. The DRWT 21, 24 25 uses a secondary smaller rotor in front of the main rotor to efficiently harness the energy from the airflow passing through the blade root region. Additionally, the DRWT can enhance flow entrainment in the turbine wake, leading to faster wake mixing and reduced wake losses 20,21,32. It should be emphasized that the inverse design methodology presented in this paper is general enough to readily work for turbines with arbitrary number of rotor disks and is not limited to DRWTs.

\section{Computational Model}

\section{Flow Solver}

The incompressible, Reynolds Averaged Navier-Stokes (RANS) equations are solved using the semi-implicit method for pressure linked equations (SIMPLE) algorithm [22]. The governing equations are

$$
\begin{aligned}
\frac{\partial \bar{u}_{i}}{\partial x_{i}} & =0, \text { and } \\
\bar{u}_{j} \frac{\partial \bar{u}_{i}}{\partial x_{j}} & =-\frac{1}{\rho} \frac{\partial \bar{p}}{\partial x_{i}}-\nu \frac{\partial^{2} \bar{u}_{i}}{\partial x_{j}^{2}}-\frac{\partial \overline{u_{i}^{\prime} u_{j}^{\prime}}}{\partial x_{j}}+\frac{f_{i}}{\rho} .
\end{aligned}
$$

The two-equation $k-\epsilon$ turbulence model by Launder and Spalding [15], with modifications suggested in Hargreaves and Wright [12, is used for turbulence closure. The source term $f_{i}$ in the momentum equation, Eq. (1) is used to model the force exerted by the turbine rotor blades on the fluid. This force is determined using the blade element theory, which requires local flow velocity at turbine location, geometric information about rotor blades such as chord and twist, and blade aerodynamic characteristics. Blade aerodynamic characteristics are specified as sectional lift and drag coefficients (airfoil polars), and are provided by the user as look-up tables. The airfoil polars may be corrected for rotational and dynamic stall effects. The body force, $f_{i}$ is calculated using the Actuator Disk Method (ADM) by distributing the force over a disk 
surrounding the turbine rotor using a Gaussian distribution [19. The numerical analysis procedure has been implemented in the unstructured, finite volume solver simpleFOAM (part of OpenFOAM) and has been previously validated against experimental data $23,24,28$.

\section{Simulation Set-up}

The objective of the paper is to demonstrate an inverse blade design procedure that uses computational fluid dynamics (CFD) for aerodynamic analysis. To minimize design time, a cost-effective computational setup is selected that is consistent with traditional inverse design methods. Specifically, a uniform, smooth inflow is considered, all rotor blades are assumed to be identical, and only mean (time-steady) performance is investigated. With these approximations, the problem becomes axisymmetric and makes the large number of calculations, as required by inverse design procedures, conducive.

Figure 1 shows an isometric view of an example axisymmetric mesh used in the study with the $x$ axis as the symmetry axis. The radius of the disk is along the $z$-direction. The lengths are nondimensionalized by the tip radius of the turbine rotor, $R$. The domain is $20 R$ in the $x$ direction and $6 R$ in the $z$ direction. It is one cell thick in the the $y$ direction and the angle between the side planes is selected to be $1^{\circ}$. The turbine rotor (or two rotors for a DRWT) is located around $x=0$ and the mesh is refined in the region of the rotor disk to capture the large gradients expected there; the mesh is also refined at the radial location corresponding to the blade tip position $(z=1)$.

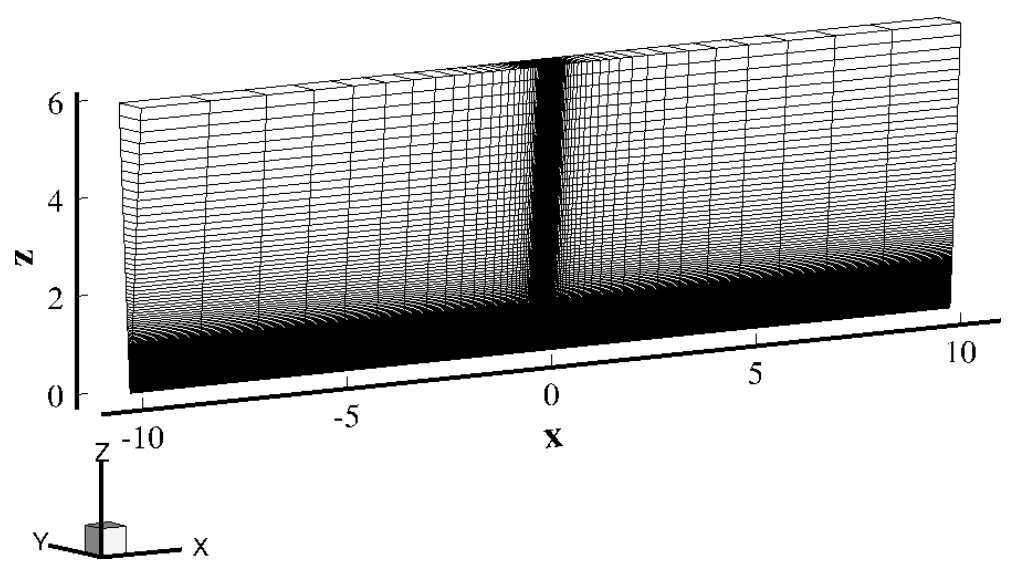

Figure 1. Isometric view of the computational mesh used for the proposed inverse design.

The inflow is in the positive $x$ direction and the force exerted by the turbine on the fluid is applied in the negative $x$ direction. For a rotor spinning clockwise, as viewed from upstream, the torque force on the fluid is applied in the positive $y$ direction. Axisymmetry boundary condition is applied on the side planes. Zero gradient is imposed for pressure and velocity at the outlet boundary. At the inlet, a zero-gradient pressure and a fixed-value velocity boundary conditions are prescribed. The same setup was used in previous studies 23, 24, 28 where the methodology was verified against experimental data for aerodynamic performance prediction.

\section{Mesh Sensitivity Study}

Sensitivity of the RANS/ADM solver to mesh size is investigated with four different meshes. Table 1 lists the mesh dimensions and the corresponding computed turbine 
power coefficient, $C_{P}=2 P / \rho u_{\infty}^{3} \pi R^{2}$, where $P$ is the power extracted by the turbine, $\rho$ is air density, $u_{\infty}$ is freestream flow speed, and $R$ is turbine rotor tip radius.

Variation with mesh size of radial distributions of angle of attack $(\alpha)$ and axial induction factor $(a)$ are plotted in Fig. 2. As seen in the figure, refining the grid beyond $N_{x} \times N_{z}=101 \times 229$ (Mesh 2) does not adversely impact the results; the $C_{P}$ becomes constant and the variations in radial distributions are minimal. Hence, the grid

resolution of Mesh 2 is selected to obtain the results presented in this paper. This study is conducted for a conventional single-rotor wind turbine and grids for multi-rotor wind turbines are deduced from this assessment. A detailed mesh sensitivity study for this solver has been reported previously in Ref. 30 .

Table 1. Mesh sensitivity study: grid dimensions and aerodynamic power coefficient.

\begin{tabular}{c|c|c}
\hline \hline Mesh \# & $N_{x} \times N_{z}$ & $C_{P}$ \\
\hline \hline 1 & $79 \times 141$ & 0.483 \\
2 & $101 \times 229$ & 0.485 \\
3 & $117 \times 279$ & 0.485 \\
4 & $136 \times 329$ & 0.485 \\
\hline \hline
\end{tabular}

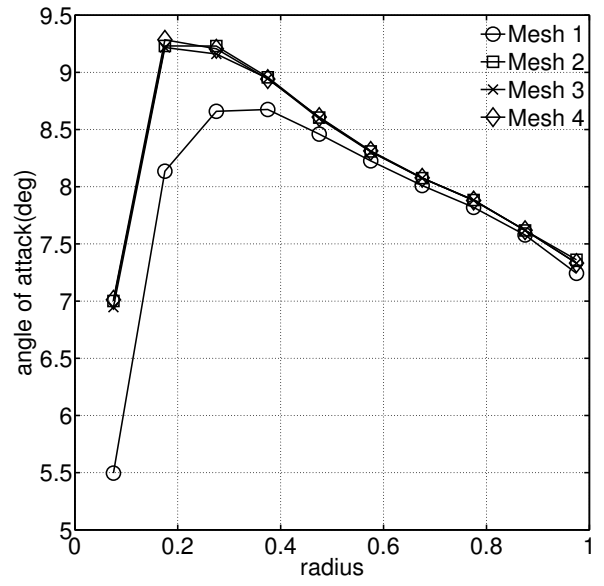

(a) angle of attack

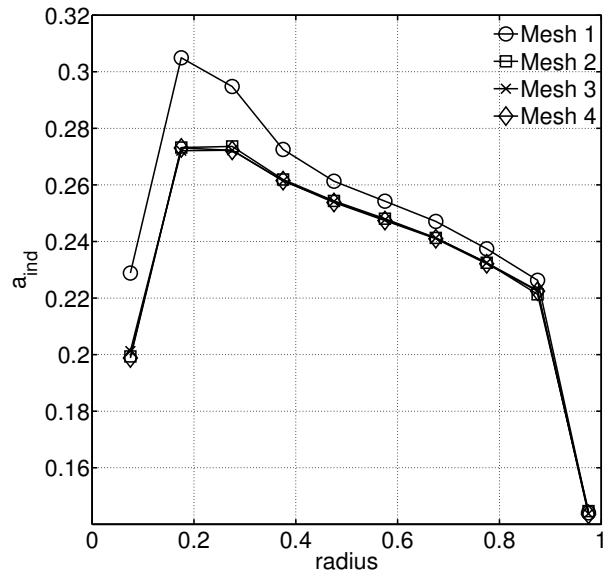

(b) axial induction factor

Figure 2. Results of the mesh sensitivity study. RANS/ADM predicted distributions of angle of attack $(\alpha)$ and axial induction factor $(a)$ are compared for four different mesh sizes.

\section{Solution Algorithm}

The purpose of using an inverse scheme in a wind turbine blade design process is to directly compute blade geometries which give the desired aerodynamic performance. The designer can choose the target aerodynamic performance parameters and prescribe their desired values (distributions). If the prescribed values of the aerodynamic performance parameters are physically consistent and achievable, the inverse design process should give the turbine blade geometry that meets the desired performance.

While there are multiple ways in which the inverse design problem can be posed, we choose to prescribe distributions of axial induction factor $(a)$, and angle of attack $(\alpha)$ as desired outcomes. As an example, in an ideal single-rotor turbine, the desired $a$ could 
be $1 / 3$ based on the $1-\mathrm{D}$ momentum theory, and the target $\alpha$ values could be selected to give the highest lift-to-drag ratio for the airfoil at that radial location. In other studies, $c_{l}$ distributions, as opposed to $\alpha$ distributions have been prescribed [17]. There is one potential problem with that choice - the $\alpha-c_{l}$ curve is multi-valued, i.e., a given value of $c_{l}$ can occur for multiple $\alpha$ values (pre- and post-stall). While at a typical design point, all radial locations are in the pre-stall region, an iterative design procedure can have transients in the post-stall region and hence the duality of $\alpha-c_{l}$ curve can pose problems. Prescription of $\alpha$ is still based on the desired $c_{l} / c_{d}$ (presumably where it is maximum), but it ensures that the turbine is not designed to operate in the post-stall regime of that airfoil. Therefore, in this study the desired parameters are set to be $\alpha$ and $a$. The inverse design algorithm then proceeds as follows:

1. The blade is discretized along the span into $m$ segments and the desired values of $\alpha$ and $a$ are prescribed for each segment $j$ as a 1-D vector $\mathbf{b}=\left\{\alpha_{j}, a_{j}\right\}^{T}$.

2. For the first iteration $(n=0)$, an initial guess of the blade geometry is provided as a 1-D vector, $\mathbf{x}_{0}=\left\{c_{j}, \theta_{j}\right\}^{T}$ of length $2 m$, where $c_{j}$ and $\theta_{j}$ are the blade chord and twist angle at segment $j$.

3. The inverse subroutine is invoked (details provided in Sec. ):

(a) With $\mathbf{x}_{n}$ as the baseline design, the direct solver is called $2 m+1$ times, to measure the effect of perturbing (by an infinitesimal amount) each element of $\mathbf{x}_{n}$ on $\mathbf{b}_{n}$ (distributions of $\alpha$ and $a$ at iteration $n$ ). The Jacobian (sensitivity) matrix $(=\partial\{\alpha, a\} / \partial\{\theta, c\}$, see Fig. 4 is calculated using first order, forward finite differences.

(b) A new estimate of the blade geometry $\mathbf{x}_{n+1}$ is computed using the Jacobian matrix and the Trust-Region-Reflective method (described in Sec.) with the aim of minimizing the difference between the desired and the computed distributions of $\alpha$ and $a$.

(c) The direct solver (RANS/ADM) is used to evaluate the aerodynamic performance of the new turbine geometry $\left(\mathbf{x}_{n+1}\right)$ and obtain $\mathbf{b}_{n+1}=\left\{\alpha_{j}, a_{j}\right\}^{T}$.

(d) If the $\boldsymbol{l}^{2}$ norm of the difference between the desired and computed aerodynamic performance $\mathbf{b}\left(=\left\{\alpha_{j}, a_{j}\right\}^{T}\right)$ is within the desired tolerance (this is just one of many stopping criteria), the algorithm is terminated and the current blade geometry $\mathbf{x}_{n+1}$ is output as the final blade design. Otherwise, the algorithm returns to step 3(a) and the iterations continue with the iteration counter $n$ incremented to $n+1$.

The algorithm is presented as a flowchart in Fig. 3.

\section{Inverse Solver}

This section provides a brief introduction to the selected inverse solver, the Trust-Region-Reflective (TRF) method. Suppose the minimum of a function $f$ is sought in a bounded or unbounded domain. As a first guess, a point $x_{0}$ in the domain is randomly picked and the function $f$ is evaluated at $x_{0}$. The essential idea behind this method is to approximate $f$ with a simpler function $q$ (see Eq. 2) that behaves similarly to $f$ in the vicinity (trust region, $N$ ) of $x_{0}$ and find a new point $x_{1}$ in $N$ where $q$ is minimum. Now if the reduction in the approximated function $(q)$ is also inherited by the original function $f, x_{1}$ is accepted as an updated estimate of the location of minimum value of $f$, and the procedure is repeated. Otherwise the trust region $(N)$ is reduced in size and the search for minimum $q$ is repeated. In order to reduce the 


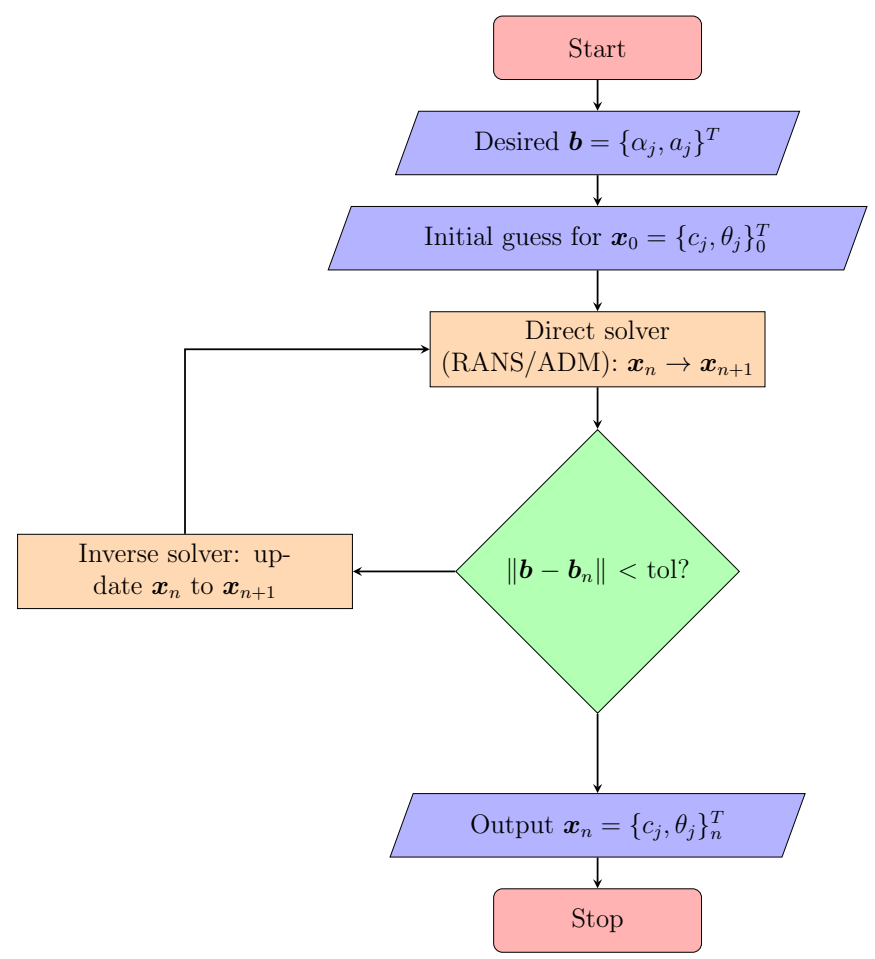

Figure 3. Flowchart of the inverse design algorithm.

computational time, the trust-region sub-problems are confined to two dimensional sub-spaces $S$. In fact, the sub-problem is defined as

$$
\arg \min \{q(s)\}, \text { subject to }\|D s\| \leq \Delta \text { and } s \in \operatorname{span}\left[s_{k}^{U}, s_{k}^{F S}\right],
$$

where $q(s)=f\left(x_{k}\right)+s^{T} g+\frac{1}{2} s^{T} H s$ is the approximation for $f$ around $x_{k}, g$ is the gradient of $f$ at $x_{k}$, and $H$ is the Hessian matrix (symmetric matrix of second derivatives of $f$ ), $D$ is the diagonal scaling matrix, $\Delta$ is the radius of the trust region, $s_{k}^{U}$ is the steepest descent direction given by $s_{k}^{U}=-g\left(g^{T} g\right) /\left(g^{T} H g\right)$, and $s_{k}^{F S}$ is either an approximate Newton direction, $H \cdot s_{k}^{F S}=-g$, or a direction of negative curvature, $\left(s^{F S}\right)_{k}^{T} \cdot H \cdot s_{k}^{F S}<0$. This formulation results in global convergence through the steepest descent or negative curvature direction while achieving a fast local convergence, when it exists, using the Newton step 4,5 in each trust region.

In this study, the multi-dimensional function $\mathbf{F}$ is defined as

$$
\mathbf{F}(\mathbf{x})=\mathbf{b}^{d}-\mathbf{b}_{n}^{c}=\left[\begin{array}{c}
F_{1}(\mathbf{x}) \\
F_{2}(\mathbf{x}) \\
\vdots \\
F_{m}(\mathbf{x}) \\
F_{m+1}(\mathbf{x}) \\
\vdots \\
F_{2 m}(\mathbf{x})
\end{array}\right]=\left[\begin{array}{c}
\alpha_{1}^{d}-\alpha_{1}^{c} \\
\alpha_{2}^{d}-\alpha_{2}^{c} \\
\vdots \\
\alpha_{m}^{d}-\alpha_{m}^{c} \\
a_{1}^{d}-a_{1}^{c} \\
\vdots \\
a_{m}^{d}-a_{m}^{c}
\end{array}\right],
$$

where superscripts $d$ and $c$ stand for the desired and calculated values respectively, and $\mathbf{b}$ is the vector of design parameters. There are multiple stopping criteria for this algorithm: maximum number of inverse iterations, minimum values of the target function, magnitude of change in the independent variables, and norm of gradient of the target function. The algorithm stops when any of these criteria is met. Based on the 
preliminary tests, the maximum number of inverse iterations is set to 30 and the minimum values of $\|\mathbf{F}\|,\|\Delta \mathbf{x}\|$ and $\|\Delta \mathbf{g}\|$ are set to $10^{-8}$. The discussions on the merits of the TRF method over other optimization schemes, details of how the algorithm proceeds under different circumstances, and determination of the size of domain $N$ for the TRF algorithm can be found in Refs. [4,5]. The interested reader is referred to Refs. 4, 5, 7] for a detailed description of the method.

\section{Verification of Inverse Design Methodology}

The proposed inverse design procedure is tested for three conventional, single rotor wind turbines (SRWTs) A description of these test cases and the performance of the inverse design procedure are presented in this section. The purpose of these tests is to ensure that the algorithm is capable of obtaining blade designs which satisfy different design target values for blades made of different airfoils. It should be noted that the direct solver (RANS/ADM model) utilized in this study has been validated previously 24,28 for wind turbine aerodynamic performance prediction. For optimization, the nonlinear least-square solver package, scipy.optimize, available with the Python scripting language is employed.

\subsection{Test Case 1: Single-Rotor Betz Optimum Turbine}

As a first test case, we attempt to design the Betz optimum rotor. Per the 1-D momentum theory, the axial induction factor should be $1 / 3$ over the entire rotor disk to achieve maximum $C_{P}$. The turbine blade is desired to be designed using one airfoil (the $18 \%$ thick DU-96-W180 airfoil) for the entire blade span. This airfoil has a high lift-to-drag ratio and typically is used for tip sections of utility-scale turbine rotor blades 24]. To achieve the best performance, the desired $\alpha$ is selected to be 10 degrees, which is where $c_{l} / c_{d}$ is maximum for the DU-96-W180 airfoil. Therefore,

$\alpha_{j}=10^{\circ}, a_{j}=1 / 3$ is specified for all radial segments $\forall j \in[1, m]$. The design tip speed ratio, $\lambda=\Omega R / u_{\infty}$ is set to be 7.0 .

The design algorithm needs to compute the Jacobian matrix $\left(=\partial\left\{\alpha_{j}, a_{j}\right\} / \partial\left\{\theta_{k}, c_{k}\right\}\right)$ in order to find the new minimum point at each iteration [5]. Information about the Jacobian matrix is also useful to understand how output variables, $\left\{\alpha_{j}, a_{j}\right\}$ at the $j^{\text {th }}$ radial segment of the blade, are influenced by change in input variables $\left\{c_{k}, \theta_{k}\right\}$ at the $k^{t h}$ radial location on the blade; $\forall j, k \in[1, m]$.

The elements of the Jacobian matrix are calculated using a forward finite difference formula. Figure 4 plots the Jacobian matrix, split in four blocks. The blade is discretized into $m=10$ segments, hence each block has $10 \times 10$ elements. Each block of the Jacobian matrix in Fig. 4 is expected to be diagonally dominant, as a change in geometry at a given radial location has maximum effect on the aerodynamic performance at the same location. The effect can be felt at nearby radial stations as well (off-diagonal terms), but it is much smaller than the diagonal terms. The results in Fig. 4 validate this hypothesis. It should be noted that due to the non-linearity of the function $\mathbf{F}(\mathbf{x})$ in Eq. (3), the Jacobian matrix needs to be updated at each optimization iteration. Figure. 4 plots the Jacobian matrix at the last iteration when convergence is achieved.

Figure 5 shows the input and output of the optimization algorithm. Radius and chord are non-dimensionalized by the tip radius $(R)$. The initial estimates for both chord and twist are uniform everywhere and are far from the final distribution. The method converges to the desired values of $\alpha$ and $a$. The $c$ and $\theta$ distributions of the converged result (see Fig. 5p are typical of wind turbine blades and the calculated values of $a$ and $\alpha$ are almost identical to the desired (prescribed) values (see Fig. 5). 


\begin{tabular}{|c|c|}
\hline$\frac{\partial \alpha}{\partial \theta}$ & $\frac{\partial \alpha}{\partial c}$ \\
\hline$\frac{\partial a}{\partial \theta}$ & $\frac{\partial a}{\partial c}$ \\
\hline
\end{tabular}

(a) Schematic

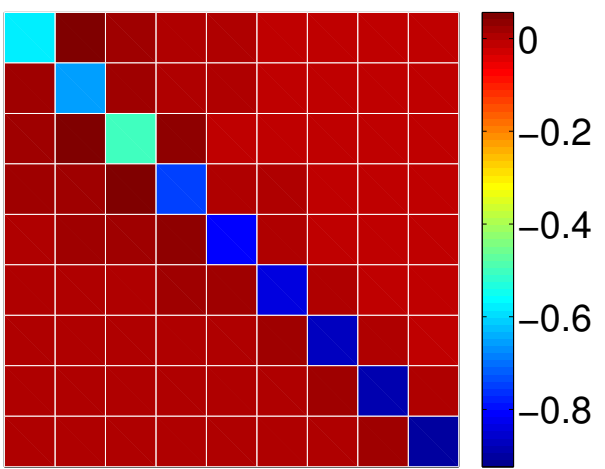

(b) $\partial \alpha / \partial \theta$

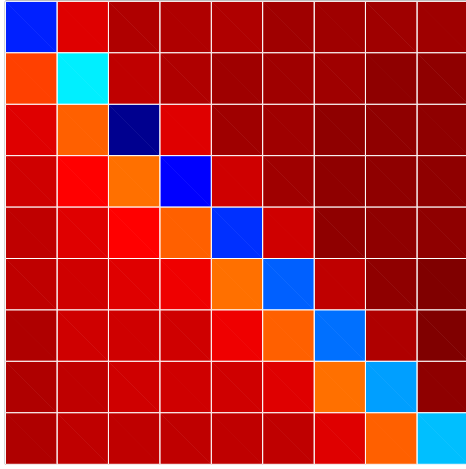

(d) $\partial a / \partial \theta$

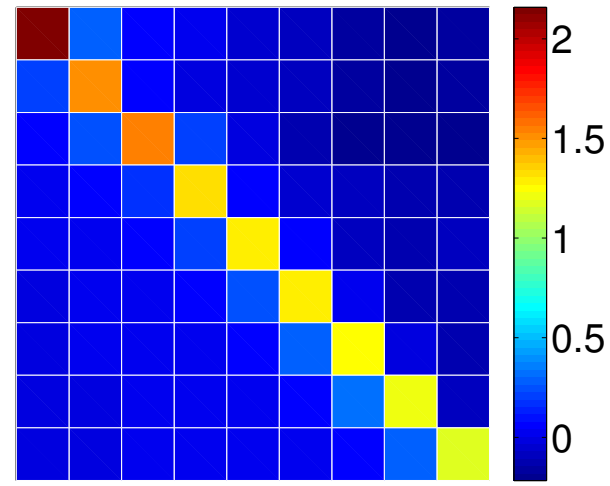

(c) $\partial \alpha / \partial c$

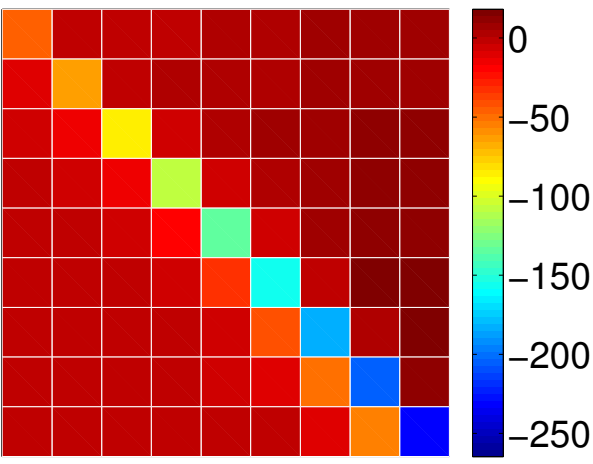

(e) $\partial a / \partial \theta$

Figure 4. Visualization of the Jacobian matrix: (a) a schematic showing the arrangement of the four different blocks in the Jacobian matrix, (b,c,d, \& e) contour plots for each of the four blocks of the matrix. The contour levels are different in each block.

The algorithm was tested with various initial input distributions of $c$ and $\theta$ and was found to always converge, demonstrating that the method is robust and insensitive to initial estimates. This test case validates the capability of the algorithm to perform inverse design of single rotor wind turbines.

\section{Test Case 2: SRWT with $\{\alpha, a\}$ Prescribed using RANS/ADM}

The objective of this test case is to verify the inverse algorithm for a turbine blade that is made of different airfoils along its span. The NREL 5 MW wind turbine 13 is considered for this test. This turbine rotor blade has cylindrical cross-section at its root and the rest of the blade is made of seven different airfoils. For this test case, the turbine geometry as given in Ref. 13 is used with the RANS/ADM solver to obtain $\alpha$ 

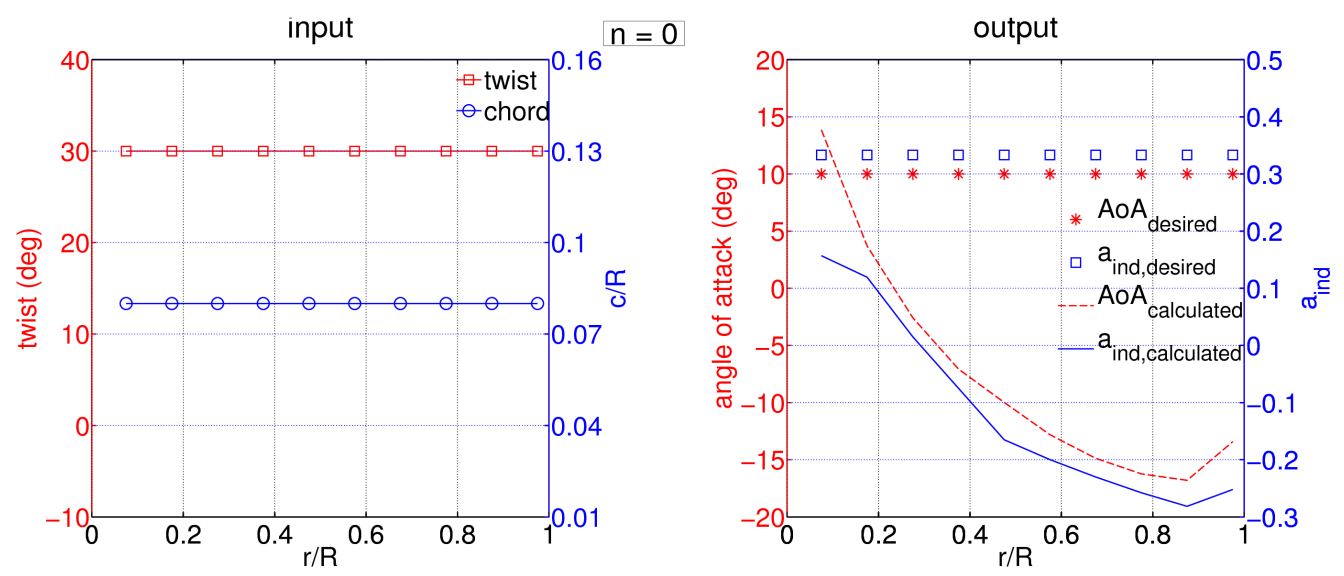

(a) At first iteration
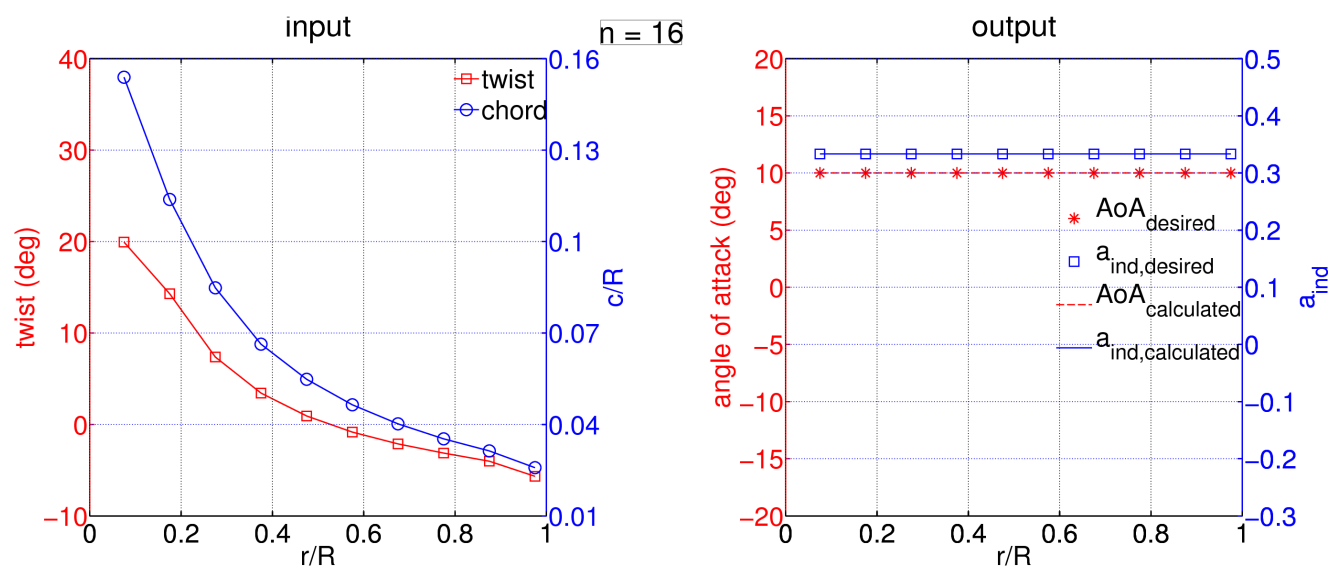

(b) At final iteration

Figure 5. Results for Test Case 1: input geometry ( $c$ and $\theta$ distributions) and output aerodynamic performance ( $\alpha$ and $a$ distributions) at the first iteration (top two plots) and final iteration (bottom two plots).

and $a$ distributions. These distributions are then prescribed as the desired values for the inverse design algorithm. Constant $c$ and $\theta$ along the blade are used as the initial guess of the blade geometry. The test of the inverse algorithm is in obtaining the original $c$ and $\theta$ distributions of Ref. [13].

The inverse algorithm can successfully reproduce the $c$ and $\theta$ distributions with less than $1 \%$ error in $\boldsymbol{l}^{2}$ norm after only 21 iterations. Figure 6 shows the original $c$ and $\theta$ distributions as well as the converged results, which are nearly overlaid. The initial guess of uniform $c$ and $\theta$ distributions are omitted from Fig. 6 for clarity.

\section{Test Case 3: SRWT with $\{\alpha, a\}$ Prescribed using BEM}

The proposed inverse design process is applied to a conventional SRWT made entirely from a single airfoil (DU-96-W180). The test is similar to Test Case 2 with the exception that BEM is used as the direct solver instead of RANS/ADM to obtain the desired distributions of $\alpha$ and $a$. Note that the inverse design algorithm remains unchanged and it still uses RANS/ADM as its direct solver.

Due to the differences between RANS/ADM and BEM algorithms, the blade design obtained using the inverse method cannot be expected to yield exactly the original 

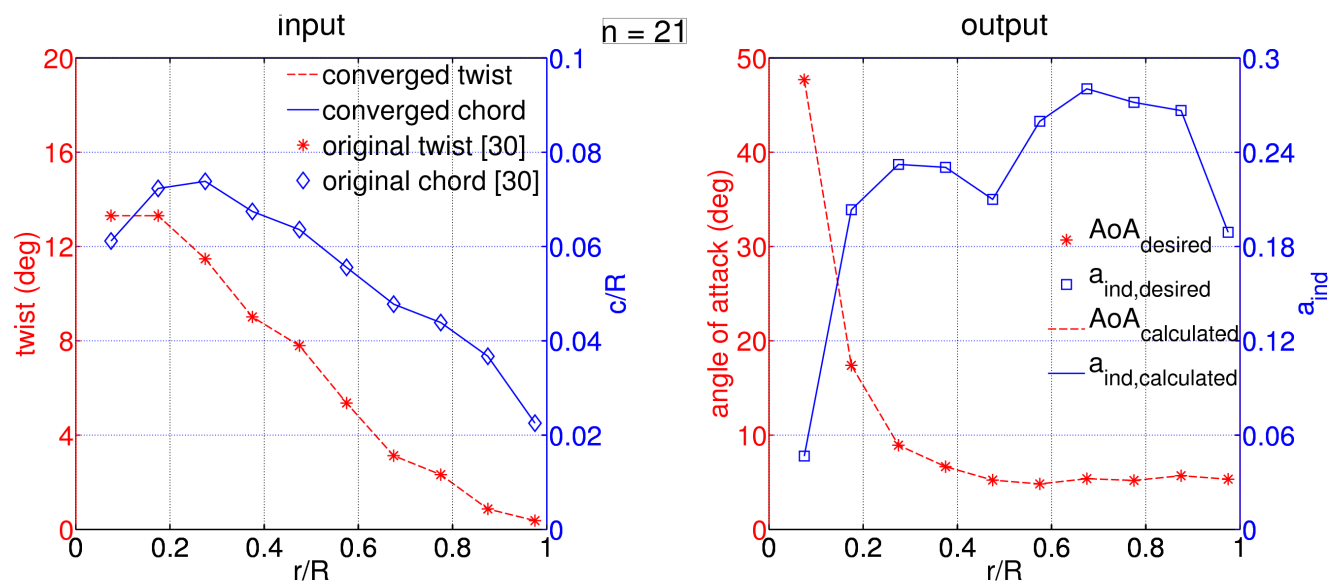

Figure 6. Results for Test Case 2: Lines show converged geometry $(c$ and $\theta)$ and converged $\alpha$ and $a$ distributions at the final iteration; symbols show original geometry from Ref. 13 and its aerodynamic performance ( $\alpha$ and $a)$ obtained via direct analysis using RANS/ADM.

geometry ( $c$ and $\theta$ distributions) as was the case in Test Case 2. The purpose of this test is to ensure the capability of the presented inverse design algorithm to yield a geometric design that is consistent with the direct solver used; in this case RANS/ADM when the desired output is prescribed by another direct solver.

Figure 7 shows the final results for this test. While the uniform initial distributions for $c$ and $\theta$ are far from the final design, the inverse design algorithm can successfully yield a blade geometry ( $c$ and $\theta$ distributions) that results in the desired distributions of $\alpha$ and $a$ over the blade span.
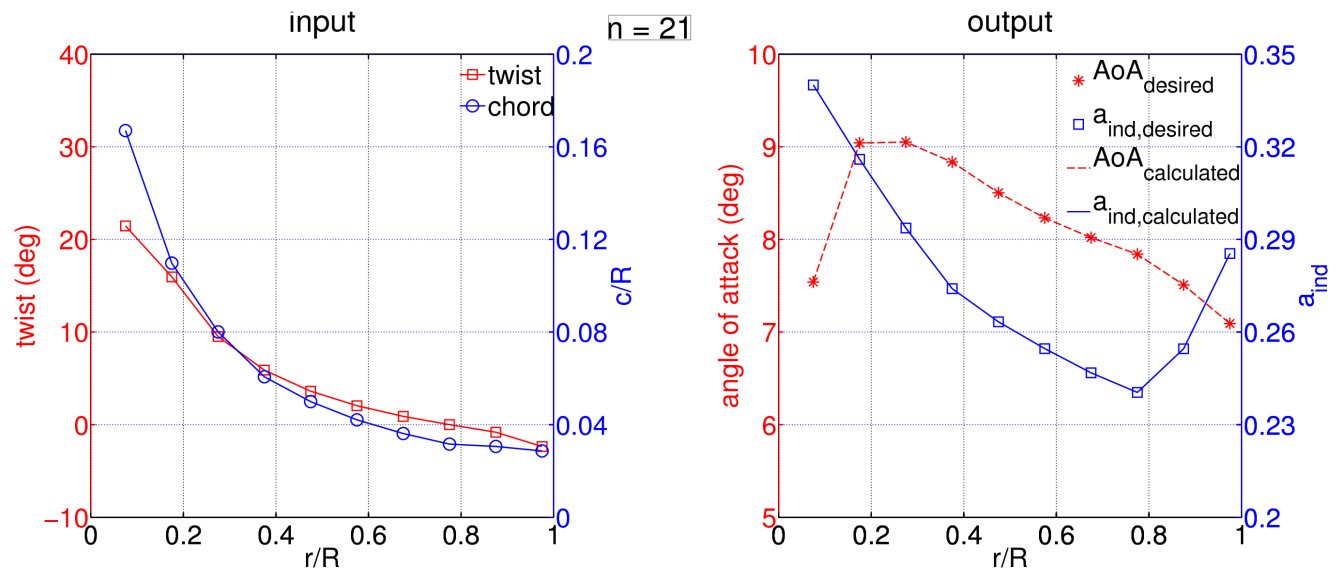

Figure 7. Results for Test Case 3: Converged $c$ and $\theta$ distributions (left) and $\alpha$ and $a$ distributions (right) at the final iteration. Predicted $\alpha$ and $a$ distributions (lines) are compared with desired values (symbols) in th right plot to show convergence.

\section{Trust-Region-Reflective Method versus Multi-dimensional Newton Iteration}

The Trust-Region-Reflective (TRF) method is compared against the multi-dimensional Newton iteration method for test cases 1 and 3. The multi-dimensional Newton iteration method is a standard optimization method that has been used in other wind 
turbine inverse design approaches (see e.g., Refs. [17,27]). The variation with iteration number of the $\boldsymbol{l}^{2}$ norm of the objective function $\boldsymbol{F}$, which is representative of residual error, is compared between the two methods. It should be noted that in order to ensure a physically realistic blade shape, the blade chord is constrained to have a value between $0.01 R$ to $0.2 R$.

As demonstrated in Fig. 8, the decrease in the norm of $\boldsymbol{F}$ for Test Case 1 is much faster with the TRF method than with the multi-dimensional Newton iteration method. In this case, the Newton method is trying to push the blade chord at some radial stations beyond the specified constraint of $0.2 R$. To impose the constraint, the chord is limited to $0.2 R$ at each iteration. This results in oscillations in the $\boldsymbol{l}^{2}$ norm of $\boldsymbol{F}$.

When the chord values do not hit a constraint during convergence, as is the case in Test Case 3, the Newton method converges faster than the TRF method. In general, the Newton method converges faster for unconstrained problems while the TRF method is found to be more stable in constrained blade design problems. The reason for this additional stability of the TRF method can be attributed to the gradual expansion of the trusted region in which the solution to the sub-problem is sought (see Section ). Nonetheless, both methods converge, and ultimately yield identical $c$ and $\theta$ distributions.

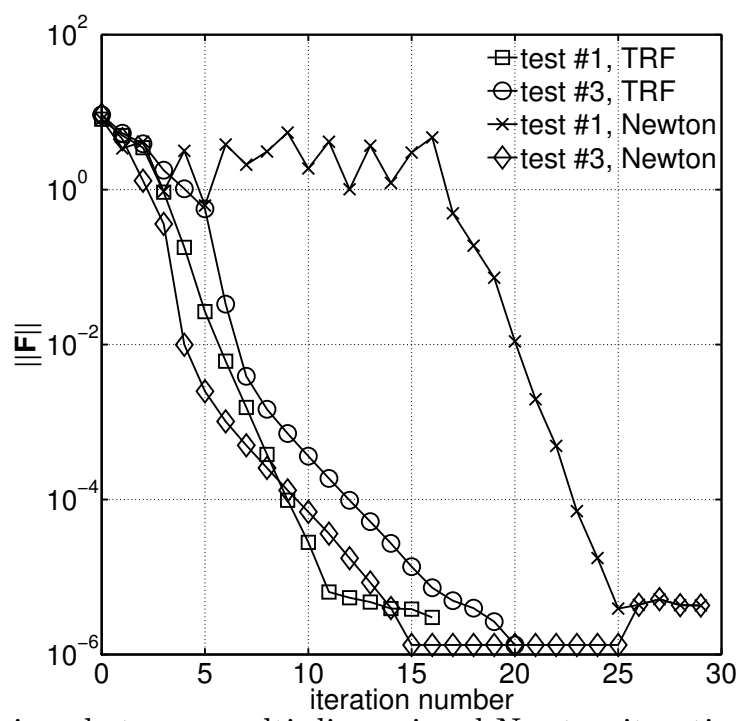

Figure 8. Comparison between multi-dimensional Newton iteration and Trust-RegionReflective (TRF) optimization methods.

\section{Extension to Multi-Rotor Wind Turbines}

This section presents the extension of the inverse blade design process to dual-rotor wind turbines (DRWTs). The procedure can be easily extended to multi-rotor turbines with more than two rotors by applying the same idea presented here.

For inverse design of DRWTs, modifications in computational set-up as well as arrangement of the Jacobian matrix are made. As mentioned in Section ??, DRWTs use a smaller, secondary rotor upstream of the main rotor in order to reduce blade root loss and enhance momentum entrainment. Two cases of DRWTs with different blades and rotor radius ratio, $R_{u} / R_{d}$ are presented in this section where subscripts $u$ and $d$ stand for upstream and downstream rotors, respectively. Upstream rotor sits at $x_{u}=-S e p / 2$ and downstream rotor is located at $x_{d}=+S e p / 2$ where $S e p$ is the rotor-rotor separation distance between the two rotors. Both rotors are modeled as actuator disks 
and the mesh is refined in the vicinity of the two rotor disk locations in both $x$ and $z$ directions. Independent variables are $\theta$ and $c$ distributions, and target parameters are radial profiles of $\alpha$ and $a$ for both rotors. For DRWTs, the Jacobian (sensitivity) matrix not only includes the effects of changing design variables of each rotor on its own target variables, but also interaction effects between the rotors. A schematic of the Jacobian matrix is shown in Fig. 9. For DRWTs, it has 4 quadrants and each quadrant has 4 blocks. The diagonal quadrants model self-influence of each rotor, while the off-diagonal quadrants model rotor-rotor interaction effects.

\begin{tabular}{|l:l|l:l|}
\hline$\frac{\partial(\alpha)_{u}}{\partial(\theta)_{u}}$ & $\frac{\partial(\alpha)_{u}}{\partial(c)_{u}}$ & $\frac{\partial(\alpha)_{u}}{\partial(\theta)_{d}}$ & $\frac{\partial(\alpha)_{u}}{\partial(c)_{d}}$ \\
\hdashline$\frac{\partial(a)_{u}}{\partial(\theta)_{u}}$ & $\frac{\partial(a)_{u}}{\partial(c)_{u}}$ & $\frac{\partial(a)_{u}}{\partial(\theta)_{d}}$ & $\frac{\partial(a)_{u}}{\partial(c)_{d}}$ \\
\hline$\frac{\partial(\alpha)_{d}}{\partial(\theta)_{u}}$ & $\frac{\partial(\alpha)_{d}}{\partial(c)_{u}}$ & $\frac{\partial(\alpha)_{d}}{\partial(\theta)_{d}}$ & $\frac{\partial(\alpha)_{d}}{\partial(c)_{d}}$ \\
\hdashline$\frac{\partial(a)_{d}}{\partial(\theta)_{u}}$ & $\frac{\partial(a)_{d}}{\partial(c)_{u}}$ & $\frac{\partial(a)_{d}}{\partial(\theta)_{d}}$ & $\frac{\partial(a)_{d}}{\partial(c)_{d}}$ \\
\hline
\end{tabular}

Figure 9. Schematic of the Jacobian for DRWT cases.

Aerodynamic interactions (coupling) between the two rotors of a DRWT makes the design process more complicated. The main interaction between the two rotors is due to the fact that a part of the downstream rotor operates in the wake of the upstream rotor. A change in the geometry of the upstream rotor affects its wake, and for practical rotor-rotor separation distances (small compared to rotor radius), the aerodynamic response of the downstream rotor. The downstream rotor also has a potential field which affects the aerodynamics of the upstream rotor, although this effect is expected to be much smaller compared to that due to the wake. The Jacobian matrix is helpful in visualizing, understanding, and quantifying such interaction effects.

To verify the extension of the inverse design algorithm to DRWTs, several cases corresponding to the SRWT test cases 2 and 3 were attempted. The inverse algorithm was able to successfully obtain the original distributions of $c$ and $\theta$ in all cases. Only two of these cases are reported here for brevity.

\section{Test Case 4: Inverse Design of DRWTs}

The DRWT considered here has two equal-size rotors $\left(R_{u} / R_{d}=1\right)$, both made entirely of the DU-96-W180 airfoil with rotor-rotor separation of $0.3 R_{d}$ and tip speed ratio $\lambda=7.0$. Similar to test case 3 , a different aerodynamic analysis solver is used to obtain radial profiles of $\alpha$ and $a$, which are then prescribed as the desired performance outcomes. The vortex lattice method (VLM) proposed by Rosenberg and Sharma 25 is selected as the direct solver to analyze the DRWT. The objective is to see if the inverse solver is able to obtain a design that gives the desired aerodynamic performance (in this case, obtained using another wind turbine analysis software). The final results of the inverse design are shown in Fig. 10. The inverse design algorithm is able to obtain geometries for both rotors of the DRWT that nearly satisfy the prescribed aerodynamic performance; there is a little difference between the desired and calculated aerodynamic performance, particularly axial induction, at the final iteration of the inverse algorithm. This difference is due to the constraint imposed on the blade chord; chord values are 
restricted at all radial locations for each rotor to be between 0.01 to 0.2 times the tip radius of the corresponding rotor. In this test case, chord values near the tip of the downstream rotor reach the lower limit $\left(0.01 R_{d}\right.$ in Fig. 10, bottom left) and this restricts the optimization algorithm from identically reproducing the target/desired values.
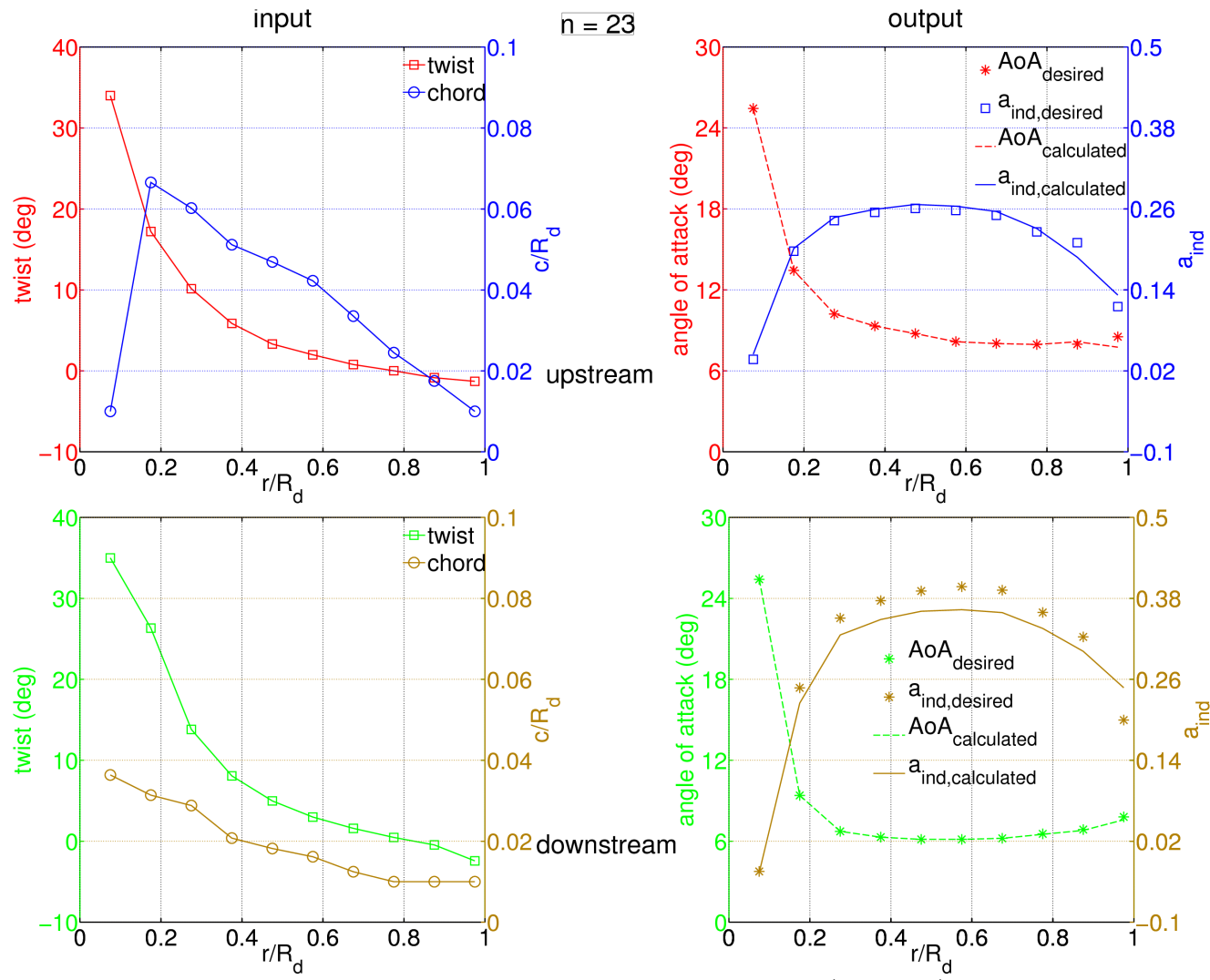

Figure 10. Results for Test Case 4: Converged geometry $(c$ and $\theta)$ and aerodynamics ( $\alpha$ and $a$ ) for upstream (top two plots) and downstream (bottom two plots) rotors. Plots on the right show desired aerodynamic performance with symbols and predicted performance of the final geometry with lines.

\subsection{Test Case 5: Inverse Design of DRWTs}

The aim of this test case is to design a more realistic DRWT which has two rotors of different sizes, and the downstream rotor has different airfoils along its span. The upstream rotor is a Betz-optimum rotor, which uses only the DU-96-W180 airfoil and the downstream rotor uses the NREL Phase VI turbine rotor blades 11]. The NREL Phase VI turbine rotor uses the S809 airfoil from $r / R \geq 0.25$ to the tip, and cylinder at its blade root region; transition sections are not included. The $2 \mathrm{D}$ polar data of the S809 airfoil at chord-based Reynolds number, $R e_{c}=5 \times 10^{5}$ were obtained using XFOIL 8. Separation between the rotors is $0.3 R_{d}$ with $R_{u} / R_{d}=0.3$ and the tip speed ratio of either rotor, defined using its tip rotor radius, is 7.0. A test case similar to Test Case 2 is performed, in which the direct solver (RANS/ADM) is evoked with a known geometry ( $c$ and $\theta$ distributions for both rotors). The RANS/ADM computed $\alpha$ and $a$ distributions for both rotor blades are then set as target values in the design process.

Initial estimates of $c$ and $\theta$ for both rotors are constant values $\left(c_{u}=0.03 R_{d}\right.$, 
$c_{d}=0.07 R_{d}$ and $\left.\theta_{u}=\theta_{d}=35^{\circ}\right)$ along the span. The converged geometry $(c$ and $\theta)$ and aerodynamic performance ( $\alpha$ and $a$ ) are presented in Fig. 11. The desired distributions of $\alpha$ and $a$ are achieved through inverse design, and the converged geometry is identical to the original geometry.
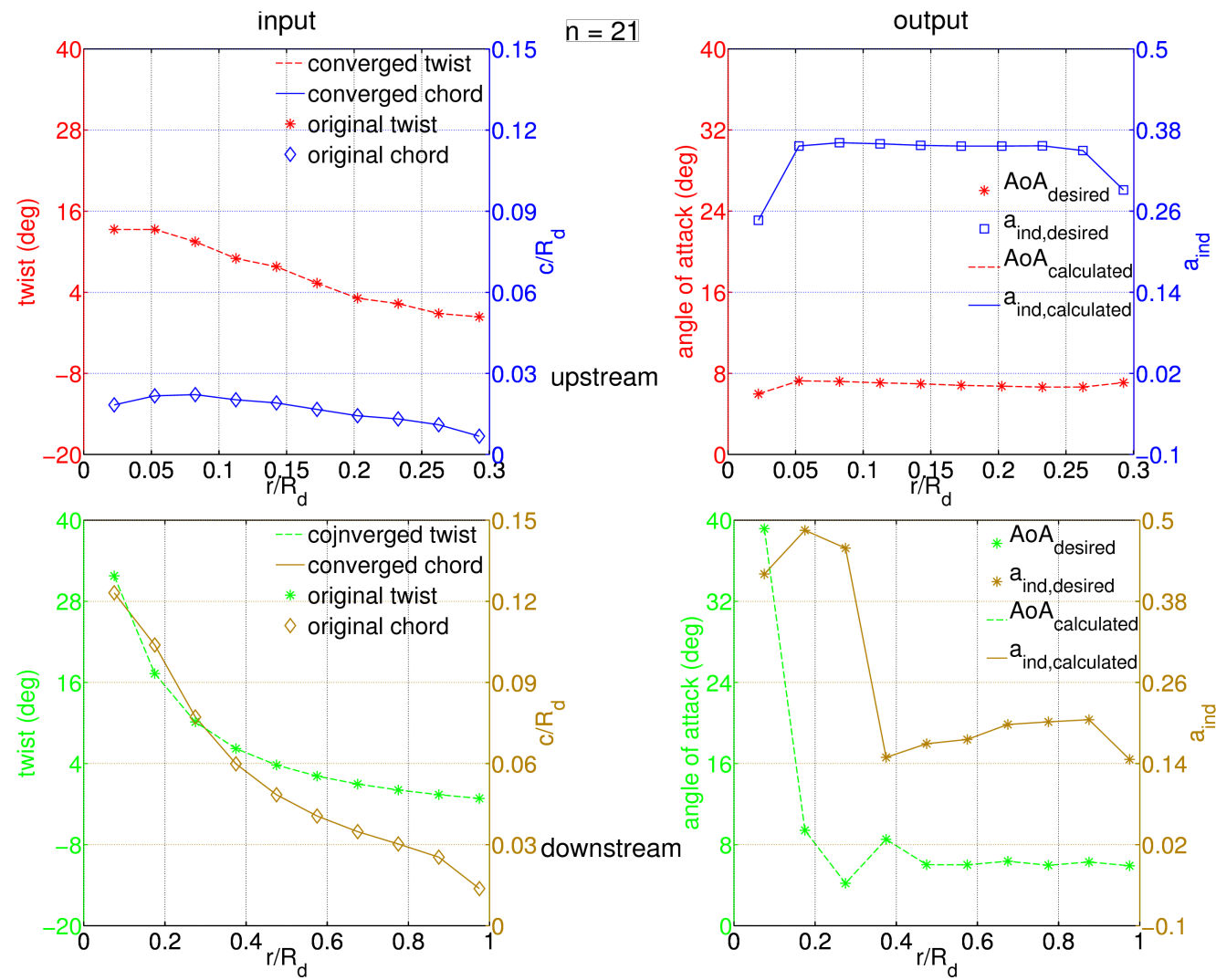

Figure 11. Results for Test Case 5: Converged geometry ( $c$ and $\theta)$ and corresponding $\alpha$ and $a$ distributions for upstream (top two plots) and downstream (bottom two plots) rotors. Symbols show original $c, \theta$ and $\alpha, a$, and lines show corresponding converged quantities.

The Jacobian at the last iteration is shown in Fig. 12. As expected, the blocks in the diagonal (upper left and bottom right) quadrants are strongly diagonal because they represent the sensitivity of output parameters to the inputs of the same rotor (refer to the schematic in Fig. 9). The upper right quadrant shows the effect of input parameters of the downstream rotor on the output of the upstream rotor. This effect is almost negligible because the rotor-rotor separation distance $\left(=0.3 R_{d}\right)$ is too large for the potential field of the downstream rotor to substantially influence the upstream rotor aerodynamics. It should be noted that the only potential field captured here is due to the aerodynamic pressure; thickness effects are not modeled. Rosenberg and Sharma 25 has shown that thickness effects can be neglected at such separation distances.

Lastly, the bottom left quadrant has non-zero components up to the tip radius of the upstream rotor $\left(R_{u} / R_{d}=0.3\right)$ since any change in the shape of the smaller upstream rotor will change the flow speed and angle in its wake and eventually the downstream rotor will be affected over a partial span. The downstream blade sections with $r>R_{u}$ (=0.3 $R_{d}$ here) will not experience much difference by changing the geometry of the upstream rotor. 

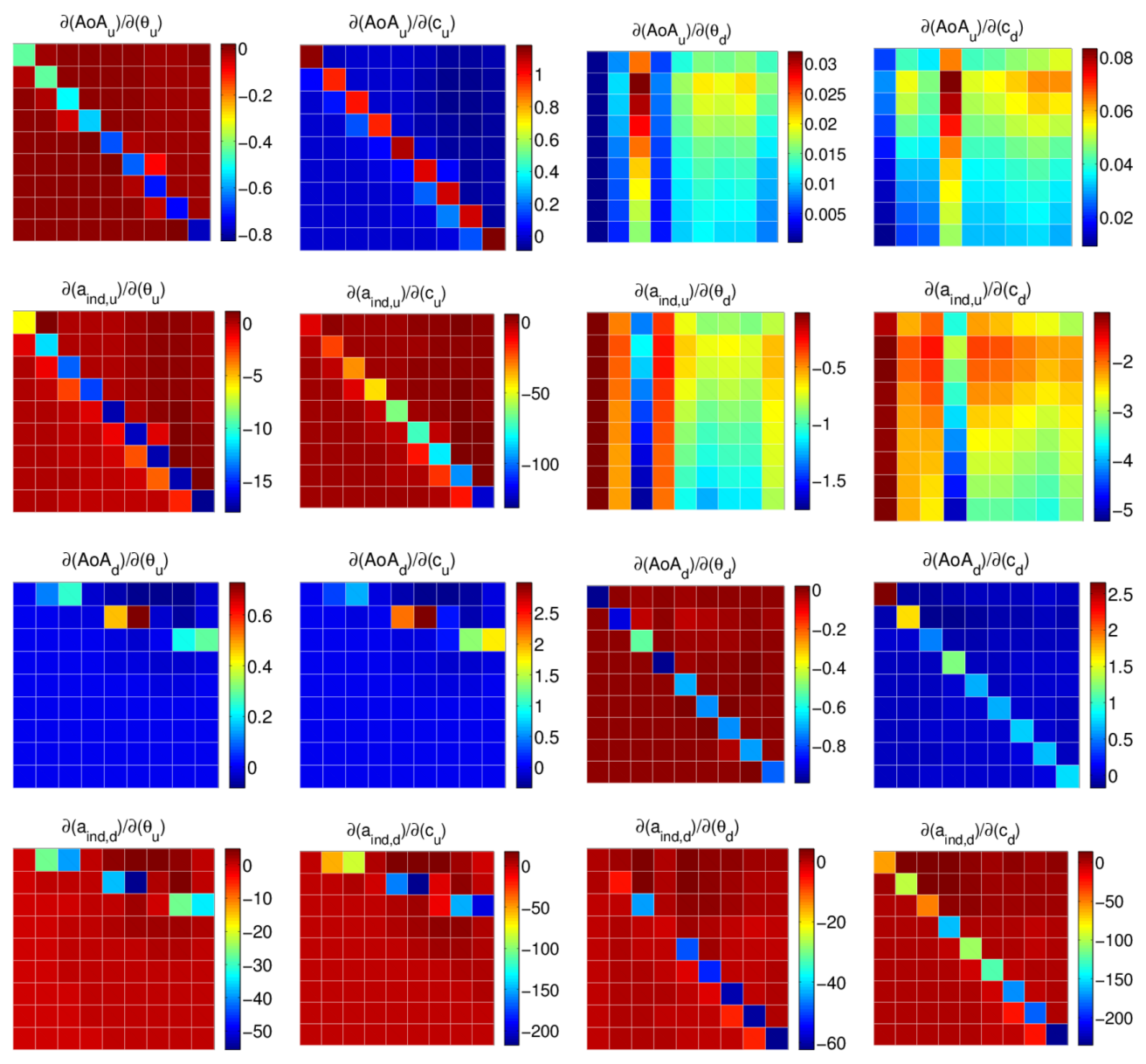

Figure 12. Different blocks of the Jacobian matrix for Test Case 5 after convergence.

\section{Conclusion}

An inverse design algorithm for wind turbine blade design is presented in this paper. The goal is to realize the desired aerodynamic performance by iteratively changing the blade geometry until convergence. The design parameters are axial induction factor and angle of attack, while the independent variables are dimensionless chord and twist distributions along the blade. The inverse design procedure requires the flow field to be solved around the wind turbine blades. In this study, RANS/ADM is chosen as the direct solver to compute wind turbine aerodynamic performance while the Trust-Region-reflective (TRF) algorithm is selected as the iterative searching method.

The design algorithm is tested with different single- and dual-rotor wind turbines. The purpose of each case is discussed and then the algorithm is put to the test to demonstrate its ability for accomplishing the design goal. In order to achieve a realistic design, constraints are applied to chord values. It is seen that when this constraint is not active (i.e. all cases except Test Case 4), the proposed inverse design algorithm converges to the blade geometry that yields the desired aerodynamic performance. However, there is a small difference between the desired and calculated values in Test Case 4 because the applied constraint does not let the procedure drive the design to minimize residual error. 
The TRF method is compared to the multi-dimensional Newton iteration method. It is found that while both methods are capable of handling the inverse problem, and converge to the same geometric distributions, they have different convergence rates for different test cases. When the design constraints come in play, the Newton method exhibits oscillations and its convergence is poor. However, when the constraints are not active, as in Test Case 3, the Newton method converges faster than TRF. In general, the TRF method was found to be more stable for constrained problems.

Another objective of this study is to extend the blade design process to multi-rotor wind turbines (DRWTs). The analysis presented in this paper demonstrates the ability of the inverse design algorithm to obtain blade geometries that satisfy the desired aerodynamic performance of DRWTs. The differences in SRWT and DRWT Jacobian matrices were discussed. The Jacobian matrix quantifies the sensitivity of the output to the input parameters. For DRWTs, it also demonstrates the aerodynamic coupling between the two rotors. The geometry of the downstream rotor blade is found to have a negligible effect on the upstream rotor for relatively large rotor-rotor separation distances. The upstream rotor however has a considerable effect on the aerodynamic performance of the downstream rotor at radial locations affected by the wake of the upstream rotor.

\section{Acknowledgments}

Funding for this work was provided by the National Science Foundation under grant number NSF/CBET-1438099. The numerical simulations reported here were performed using the NSF XSEDE resources available to the authors under grant number TG-CTS130004, and the Argonne Leadership Computing Facility, which is a DOE Office of Science User Facility supported under Contract DE-AC02-06CH11357.

\section{References}

1. C. N. Adkins and R. H. Liebeck. Design of optimum propellers. Journal of Propulsion and Power, 10(5):676-682, 1994.

2. A. Alaimo, A. Esposito, A. Messineo, C. Orlando, and D. Tumino. 3d cfd analysis of a vertical axis wind turbine. Energies, 8(4):3013-3033, 2015.

3. P. Bachant and M. Wosnik. Modeling the near-wake of a vertical-axis cross-flow turbine with 2-d and 3-d rans. arXiv preprint arXiv:1604.02611, 2016.

4. R. H. Byrd, J. C. Gilbert, and J. Nocedal. A trust region method based on interior point techniques for nonlinear programming. Mathematical Programming, 89(1):149-185, 2000.

5. R. H. Byrd, R. B. Schnabel, and G. A. Shultz. A trust region algorithm for nonlinearly constrained optimization. SIAM Journal on Numerical Analysis, 24(5):1152-1170, 1987.

6. J.-J. Chattot. Optimization of wind turbines using helicoidal vortex model. Journal of solar energy engineering, 125(4):418-424, 2003.

7. T. F. Coleman and Y. Li. On the convergence of interior-reflective newton methods for nonlinear minimization subject to bounds. Mathematical programming, 67(1-3):189-224, 1994. 
8. M. Drela. Xfoil: An analysis and design system for low reynolds number airfoils. In Low Reynolds number aerodynamics, pages 1-12. Springer, 1989.

9. P. Giguere and M. Selig. Desirable airfoil characteristics for large variable-speed horizontal axis wind turbines. Journal of solar energy engineering, 119(3):253-260, 1997.

10. S. Goldstein. On the vortex theory of screw propellers. Proceedings of the Royal Society of London. Series A, Containing Papers of a Mathematical and Physical Character, 123(792):440-465, 1929.

11. M. Hand, D. Simms, L. Fingersh, D. Jager, J. Cotrell, S. Schreck, and S. Larwood. Unsteady aerodynamics experiment phase vi: wind tunnel test configurations and available data campaigns. National Renewable Energy Laboratory, Golden, CO, Report No. NREL/TP-500-29955, 2001.

12. D. Hargreaves and N. G. Wright. On the use of the $\mathrm{k}-\varepsilon$ model in commercial cfd software to model the neutral atmospheric boundary layer. Journal of Wind Engineering and Industrial Aerodynamics, 95(5):355-369, 2007.

13. J. Jonkman, S. Butterfield, W. Musial, and G. Scott. Definition of a 5-mw reference wind turbine for offshore system development. National Renewable Energy Laboratory, Golden, CO, Technical Report No. NREL/TP-500-38060, 2009.

14. H. Lam and H. Peng. Study of wake characteristics of a vertical axis wind turbine by two-and three-dimensional computational fluid dynamics simulations. Renewable Energy, 90:386-398, 2016.

15. B. E. Launder and D. Spalding. The numerical computation of turbulent flows. Computer methods in applied mechanics and engineering, 3(2):269-289, 1974.

16. K.-H. Lee, K.-H. Kim, D.-H. Lee, K.-T. Lee, and J.-P. Park. Two-step optimization for wind turbine blade with probability approach. Journal of Solar Energy Engineering, 132(3):034503, 2010.

17. S. Lee. Inverse design of horizontal axis wind turbine blades using a vortex line method. Wind Energy, 18(2):253-266, 2015.

18. P. Marsh, D. Ranmuthugala, I. Penesis, and G. Thomas. Three-dimensional numerical simulations of straight-bladed vertical axis tidal turbines investigating power output, torque ripple and mounting forces. Renewable Energy, 83:67-77, 2015 .

19. R. Mikkelsen. Actuator disc methods applied to wind turbines. PhD thesis, Technical University of Denmark, 2003.

20. B. Moghadassian, A. Rosenberg, H. Hu, and A. Sharma. Numerical investigation of aerodynamic performance and loads of a novel dual rotor wind turbine.

21. B. Moghadassian, A. Rosenberg, and A. Sharma. Numerical investigation of aerodynamic performance and loads of a novel dual rotor wind turbine. Energies, 9(571), 2016.

22. S. V. Patankar and D. B. Spalding. A calculation procedure for heat, mass and momentum transfer in three-dimensional parabolic flows. International journal of heat and mass transfer, 15(10):1787-1806, 1972. 
23. A. Rosenberg. A Computational Analysis of Wind Turbine and Wind Farm Aerodynamics with a Focus on Dual Rotor Wind Turbines. PhD thesis, Iowa State University, 2016.

24. A. Rosenberg, S. Selvaraj, and A. Sharma. A novel dual-rotor turbine for increased wind energy capture. In Journal of Physics: Conference Series, volume 524, page 012078. IOP Publishing, 2014.

25. A. Rosenberg and A. Sharma. A prescribed-wake vortex lattice method for preliminary design of co-axial, dual-rotor wind turbines. Journal of Solar Energy Engineering, 138(6), 2016.

26. M. S. Selig and V. L. Coverstone-Carroll. Application of a genetic algorithm to wind turbine design. Journal of Energy Resources Technology, 118(1):22-28, 1996.

27. M. S. Selig and J. L. Tangler. Development and application of a multipoint inverse design method for horizontal axis wind turbines. Wind Engineering, 19(2):91-106, 1995.

28. S. Selvaraj. Numerical investigation of wind turbine and wind farm aerodynamics. $\mathrm{PhD}$ thesis, Iowa State University, 2014.

29. J. N. Sørensen and A. Myken. Unsteady actuator disc model for horizontal axis wind turbines. Journal of Wind Engineering and Industrial Aerodynamics, 39(1):139-149, 1992.

30. A. S. Thelen, L. T. Leifsson, A. Sharma, and S. Koziel. Direct and surrogate-based optimization of dual-rotor wind turbines. In 34th Wind Energy Symposium, page 1265, 2016.

31. N. Troldberg. Actuator line modeling of wind turbine wakes (ph. d. thesis). Department of Mechanical Engineering, Technical University of Denmark, Lynby, 2008.

32. Z. Wang, W. Tian, A. Ozbay, A. Sharma, and H. Hui. An experimental study on the aeromechanics and wake characteristics of a novel twin-rotor wind turbine in a turbulent boundary layer flow. Experiments in Fluids, 57(-):150, 2016. 